\title{
CRITICAL CONCENTRATION FOR THE METAL-NON METAL TRANSITION IN n-TYPE GERMANIUM AND SILICON
}

\author{
K. F. BERGGREN and B. SERNELIUS \\ Department of Physics and Measurement Technology \\ University of Linköping, S-581 83 Linköping, Sweden
}

\begin{abstract}
Résumé. - La transition métal-non métal dans Ge et Si de type n est considérée à l'aide d'un modèle qui a des traits communs avec des travaux récents sur des gouttes électrons-trous. La concentration critique est déterminée en comparant l'énergie de la phase métallique avec l'énergie d'un électron lié à un ion donneur. L'accord avec les expériences est satisfaisant et les déplàcements chimiques des niveaux sont reproduits. Des estimations précédentes des concentrations critiques sont revues.
\end{abstract}

Abstract. - The metal-non metal transition in $\mathrm{n}$-type $\mathrm{Ge}$ and $\mathrm{Si}$ is considered by means of a model that has features in common with recent work on electron-hole droplets. The critical concentration is determined by comparing the energy of the metallic phase with the energy of an electron bound to a donor ion. Agreement with experiments is satisfactory and chemical shifts are reproduced. Previous estimates of the critical concentration are reviewed.

1. Introduction. - In some non-crystalline systems in which atomic configurations are disordered in some way or another, metal-non metal (MNM) transitions are observed when appropriate physical parameters such as densities, impurity concentrations, electric fields, and temperatures are changed. Doped semiconductors, like Ge : $\mathbf{P}$ and $\mathrm{Si}: \mathbf{P}$ which we shall discuss here, are one of the best examples of such transitions. Hence they have been the subject of a large number of experimental and theoretical studies. Other examples are metal-ammonia solutions, metalrare gas mixtures, supercritical metallic fluids, and excitons of high densities [1].

At low concentrations and temperatures the conduction in doped semiconductors is due to the hopping between impurity centers. With increasing concentration, however, a rapid (but not sharp) increase in the conductivity occurs at a critical concentration, $n_{\mathrm{c}}$. For concentrations larger than $n_{\mathrm{c}}$ the system has metallic characteristics. So far, two major theoretical schemes have been put forward to explain the observed transition and the related electronic properties, i. e. the Mott-Hubbard-Anderson [1-3] scheme and the percolation theory for inhomogenous regions [4, 5].

At this conference we find it appropriate to summarize previous estimates of $n_{\mathrm{c}}$. The classical explanation of the MNM transition was given by Mott [1], who predicted that it would occur when

$$
n_{\mathrm{c}}^{1 / 3} \cdot a_{\mathrm{H}}^{*} \simeq 0.25
$$

( $a_{\mathrm{H}}^{*}$ is the characteristic Bohr radius of the material). According to Mott the metallic state collapses when an impurity ion, dielectrically screened by the conduction electrons, aquires a bound state. Although Mott's criterion is in good agreement with experiments this agreement seems to be fortuitous for the following reasons. The derivation of $n_{c}$ was based on a simple variational calculation with a 1s-function and a Thomas-Fermi screened Coulomb potential. A series of subsequent calculations [6-11] have indicated that the numerical constant in Mott's criterion is quite sensitive to the choice of bound state wave function, dielectric screening and the bandstructure of the host material (e. g. the number of conduction band minima). It therefore seems fair to say that a reliable theoretical value of the constant in Mott's criterion is not available as yet from this type of calculations. Another result is that Mott's original model predicts a sharp transition in contrast to observations.

Another line of approach is due to Hubbard [2]. On the basis of a simplified tight-binding Hamiltonian and an approximate decoupling of the Green's functions Hubbard demonstrated that a gap opens up at the Fermi level when the bandwidth $\Delta$ of the unperturbed band approximately equals the intrasite repulsion energy $U$. The Hubbard model supplements the Mott model in the sense that is emphasizes the tight binding, or atomic limit rather than the metallic one. Furthermore the MNM transition is continuous in Hubbard's model (also for crystalline systems). Some estimates of $n_{c}$ have been made by means of Hubbard's model. Using hydrogenic orbitals to compute $\Delta$ and $U$ and ignoring disorder Caron and Kemeny [12] and Berggren [13] have obtained $n_{\mathfrak{c}}^{1 / 3} a_{\mathrm{H}}^{*} \simeq 0.2$. In [13] the case of anisotropic conduction band minima were also considered. Similar calculations have recently been performed also by Bergersen et al. [14]. Closely related is also Gutzwiller's [15] 
variational approach to narrow bands as applied to $\mathrm{Si}: \mathrm{P}[16,17]$. Although some of these estimates are in good agreement with experiments one notes that an accurate evaluation of $\Delta$ and $U$ is indeed quite difficult. Hence it may be hard to tell how reliable the computed values of $n_{\mathrm{c}}$ are in the case of the Hubbard model as well. As mentioned above disorder has also been ignored in the estimates. Concluding our list of previous theoretical predictions for $n_{\mathrm{c}}$ we may mention the dielectric catastrophe occuring in the ClausiusMosotti equation [18, 19].

In the calculation of $n_{\mathfrak{c}}$, as listed above, no attempts to include the effects of disorder have been made. A current picture [1] is that the transition is primarily triggered by electron correlation but that disorder adds some important features. Thus in the Hubbard model the repulsion $U$ gradually gives rise to a pseudogap in the density of states as $n_{c}$ is approached from the metallic side. According to Mott, Anderson localization sets in first in this pseudogap. We may then distinguish between two transitions, one at which localized states appear leading to variable range hopping and another at which the Hubbard bands actually split. This would explain why the transition is not sharp. One may ask how close these transitions are, or how strongly correlated the system really is in the vicinity of the transition. Oaki and Kamimura [20] have recently studied the Hubbard model for a structurally random system. Using hydrogenic orbitals they find that the bands split at $n_{\mathrm{c}}^{1 / 3} a_{\mathrm{H}}^{*} \simeq 0.18$, which would imply that $n_{\mathrm{c}}$ is not very sensitive to whether the system is crystalline or disordered. The Anderson transition is found to set in at $n_{\mathrm{c}}^{1 / 3} a_{\mathrm{H}}^{*} \simeq 0.20$, i. e. quite close to the density at which the sub-bands split. There is also some experimental evidence that this may be the case. In the localized regime, but before band splitting takes place, there is a continuum of states at $\varepsilon_{\mathrm{F}}$ available for excitations. It therefore seems reasonable to assume that the dielectric constant would be infinite (or at least very high) in this regime and would take on finite values only when the bands split. In the measurements of Castner et al. [21] the dielectric constant of for example Si : $\mathrm{P}$ approaches infinity at

$$
n=3.5( \pm 0.4) \times 10^{18} \mathrm{~cm}^{-3}
$$

in close agreement with $n_{\mathrm{c}} \simeq 3 \times 10^{18} \mathrm{~cm}^{-3}$ obtained from conductivity data. The considerations above thus seem to support the idea that a correlation induced pseudogap is formed in the metallic phase and not much before band splitting occurs Anderson localization arises in this gap. Most recently, however, Mott [22] has come to doubt this picture and is inclined to believe that, for example, highly doped silicon is less correlated than previously thought. According to Mott a pure Anderson transition due to the random positions of the donors seems equally capable of explaining the transition. In particular it then becomes easier to understand why quite strong compensation (up to $80 \%$ ) has only a minor effect on the critical concentration. The least we can say is that we are for the moment left in total confusion about the true character of the transition!

Above we have reviewed previous estimates of $n_{c}$ and pointed out the difficulties associated with its strict prediction. In our opinion it is therefore of value to try to deal with the problem in another way. We shall therefore discuss a model that has been inspired by recent work on electron-hole droplets. (A preliminary report is found in [23]). Doing so we will not claim that our model is more accurate than previous ones. We simply believe that a delicate problem should be approached in as many ways as possible.

2. The model. - At low temperatures electrons and holes in semiconductors are bound in excitons by the Coulomb attraction between them. At high concentrations the interaction among the excitons becomes essential and leads to the formation of collective substances, electron-hole droplets. (For a review see e. g. [24]). We may understand the formation of an electron-hole plasma at $T=0 \mathrm{~K}$ in the following elementary way. If at some density the energy (per electron-hole pair) of the plasma is less than the binding energy of an exciton in the gaseous phase electronhole droplets will form spontaneously. The analogy with doped semiconductors is apparent. For n-type substances we simply let the holes be infinitely heavy. Isolated impurity atoms then play the role of excitons, and the collective state is an electron gas with the same characteristics as the conduction band of the host material. An elementary criterion for the transition is obtained by equating the energy of the metallic phase with $-I$, where $I$ is the ionization energy of an electron bound to an impurity ion. Our model is similar to the one discussed by Baltensperger [25], but differs in some important aspects ; the true band structure of the host material is incorporated as well as the variation of $I$ with density. It is worth pointing out that our model is free of any adjustable parameters.

Let us now consider the metallic phase. In the effective mass theory the Hamiltonian is

$$
\begin{aligned}
\mathcal{F}= & \sum_{i}\left\{-\frac{\hbar^{2}}{2}\left[\frac{1}{m_{t}}\left(\frac{\partial^{2}}{\partial x_{i}^{2}}+\frac{\partial^{2}}{\partial y_{i}^{2}}\right)+\frac{1}{m_{1}} \frac{\partial^{2}}{\partial z_{i}^{2}}\right]+\right. \\
& \left.+\sum_{g} \omega\left(\mathbf{r}_{i}-\mathbf{R}_{g}\right)\right\} \\
& +\frac{1}{2} \sum_{i \neq j} e^{2} / \kappa r_{i j}+\frac{1}{2} \sum_{g \neq h} e^{2} / \kappa R_{g h} .
\end{aligned}
$$

Here $m_{\mathrm{t}}$ and $m_{1}$ are the transverse and longitudinal masses, respectively, $\omega\left(\mathbf{r}_{i}-\mathbf{R}_{g}\right)$ is the interaction between a donor at site $\mathbf{R}_{g}$ and an electron at $\mathbf{r}_{i}, \kappa$ is the dielectric screening of the host material, and

$$
r_{i j}=\left|\mathbf{r}_{i}-\mathbf{r}_{j}\right|, \quad R_{g h}=\left|\mathbf{R}_{g}-\mathbf{R}_{h}\right| .
$$


In the following we shall treat the electron-donor ion interaction as a perturbation. For this purpose we should thus add and subtract a compensating uniform background. The perturbing field is therefore ( $n$ is the electron density)

$$
V(\mathbf{r})=\sum_{g} \omega\left(\mathbf{r}-\mathbf{R}_{g}\right)+e^{2} \int \mathrm{d} \mathbf{r} n / r \kappa .
$$

The total energy of the metallic phase can hence be written as (below we always mean the energy per electron-donor ion pair)

$$
E(n)=E_{\mathrm{kin}}+E_{\mathrm{x}}+E_{\mathrm{M}}+E_{\mathrm{c}}+E_{\mathrm{p}}+E_{\mathrm{BS}}
$$

where the four first contributions refer to the unperturbed uniform electron gas. If $v$ is the number of conduction band minima ( 4 and 6 for $\mathrm{Ge}$ and Si, respectively) the kinetic energy is (see e. g. [24])

$$
E_{\mathrm{kin}}=3 \hbar^{2}\left(3 \pi^{2} n / v\right)^{2 / 3} / 10\left(m_{1} m_{t}^{2}\right)^{1 / 3}
$$

and the exchange energy

$E_{\mathrm{x}}=-\gamma^{1 / 6} \sin ^{-1}(\sqrt{1-\gamma}) 3 e^{2} q_{\mathrm{F}} / 4 \pi \kappa \sqrt{1-\gamma}$

where $\gamma=m_{\mathrm{t}} / m_{1}$ and $q_{\mathrm{F}}=\left(3 \pi^{2} n / v\right)^{1 / 3}$. (As shown in [14] intervalley exchange may be omitted in $E_{\mathrm{x}}$ ).

If $g(r)$ is the pair distribution function for the donor ions the Madelung energy is

$$
\left.E_{\mathrm{M}}=\frac{e^{2}}{2} n \int \mathrm{d} \mathbf{r}[g(\mathbf{r})-1)\right] / \kappa r
$$

A simple choice of $g(r)$ is

$$
g(r)=\left\{\begin{array}{l}
1 r>R \\
0 r<R
\end{array}\right.
$$

where $R$ is of the order of the Wigner-Seitz radius of host material. The distribution of donors is thus random, except for the fact that two ions cannot occupy the same site. The Madelung energy then becomes

$$
E_{\mathrm{M}} \simeq-\pi e^{2} n R^{2} / \kappa=-3 e^{2} R^{2} / 4 \kappa r_{\mathrm{s}}^{3}
$$

where $r_{\mathrm{s}}$ is the radius of the electron sphere $\left(n=3 / 4 \pi r_{\mathrm{s}}{ }^{3}\right)$.

Obviously $R \ll r_{\mathrm{s}}$ for the present system, so one can to a very good approximation put $E_{\mathrm{M}} \simeq 0$.

The contribution $E_{\mathrm{p}}$ in eq. (3) is the first correction in perturbation theory due to $V(r)$ in eq. (2), i. e.

$$
E_{\mathrm{p}}=\int \mathrm{d} \mathbf{r} n V(\mathbf{r})=n \lim _{q \rightarrow 0}\left[\omega(\mathbf{q})+4 \pi e^{2} / \kappa q^{2}\right]
$$

where $\omega(\mathbf{q})$ is the Fourier transition of $\omega(\mathbf{r})$. To estimate the effect of this term we may use the bare pseudopotential

$$
\omega_{0}(r)=- \begin{cases}V_{0} & r<r_{0} \\ \frac{e^{2}}{r} & r>r_{0}\end{cases}
$$

which has been used by Pantelides [26] in connection with shallow donor states in Si. The potential $\omega(\mathbf{q})$ in eq. (9) equals $\omega_{0}(q)$ divided by the ( $q$-dependent) dielectric function of the host material. Eqs. (9) and (10) thus give

$$
E_{\mathrm{p}}=\frac{r_{0}^{2} e^{2}}{2 \kappa r_{\mathrm{s}}^{3}}\left[3-2 V_{0} r_{0} / e^{2}\right]
$$

To second order in perturbation theory the potential in eq. (2) gives rise to the band structure energy

$$
E_{\mathrm{BS}}=\frac{v e^{2}}{(2 \pi)^{2}} \int_{0}^{\infty} \mathrm{d} q q^{2}\left|\omega_{0}(q)\right|^{2} \chi^{0}(q) a(q) / \kappa(q) \varepsilon_{12}(q)
$$

where $\chi^{0}(q)$ is a density-density response function, $a(q)$ a structure factor related to $(7)$, and $\varepsilon_{12}(q)$ a dielectric screening function with the polarizabilities from both the electron gas and the host materials included. In $\varepsilon_{12}$ we have used the Hubbard-Shaw scheme as adjusted to the present problem. Furthermore we have replaced $m_{\mathrm{t}}$ and $m_{1}$ in $\chi^{0}(q, \omega)$ with the density of states effective mass in order to make the calculations tractable. Space will not allow us to deal with the correlation energy, $E_{\mathrm{c}}$ in detail. We therefore only mention that we have, followed the treatment of Brinkman and Rice [27], with the only difference that the contributions from the holes are removed.

In the insulating phase we shall treat the variation of the ionization with density in a simple semi-classical way (see [19] and references therein). In the presence of a large density of polarizable donors the ionization energy is shifted by the factor $(1-4 \pi n \alpha(0) / 3)^{1 / 2}$ where $\alpha(0)$ is the static atomic polarizability.

3. Results and Summary. - The energy of the metallic phase has been computed for $\mathrm{n}$-type $\mathrm{Ge}$ and $\mathrm{Si}$ with values of $m_{t}, m_{1}$, and $\kappa$ taken from [28]. The results are shown in figures 1 and 2 . In the metallic phase the central cell corrections expressed through $\omega(q)$ are evidently unimportant. The dominant contributions come from $E_{\text {kin }}$ and $E_{\mathrm{BS}}$. It is noteworthy that the inclusion of multi-valley character of the conduction bands is important for the cohesion of the metallic phase. The dashed curves in figures 1 and 2 refer to the ionization energies of bound donor electrons. In the case of doped Si they have been computed from the experimental polarizabilities given in [21], and in the case of Ge as given by the hydrogenic expression for $\alpha$. For $\mathrm{Si}: \mathrm{Sb}, \mathrm{Si}: \mathrm{P}$, and $\mathrm{Si}:$ As we thus obtain the critical densities $\sim 3 \times 10^{18}, 4 \times 10^{18}$, and $9 \times 10^{18} \mathrm{~cm}^{-3}$. The experimental values [21] are $\sim 3 \times 10^{18}$, $3.5 \times 10^{18}$, and $6.4 \times 10^{18} \mathrm{~cm}^{-3}$, respectively. For $\mathrm{Ge}: \mathrm{Sb}, \mathrm{Ge}: \mathbf{P}$ and $\mathrm{Ge}:$ As the theoretical values are $\sim 1.0 \times 10^{17}, 2.5 \times 10^{17}$, and $3.7 \times 10^{17} \mathrm{~cm}^{-3}$ to be compared with the experimental, values [1] $\sim 0.95 \times 10^{17}, 2.5 \times 10^{17}$ and $3.5 \times 10^{17} \mathrm{~cm}^{-3}$, respectively. It is indeed hard to ask for a better agreement ! In the introduction, however, we pointed out possible inaccuracies in previous estimates of $n_{\mathrm{c}}$. We 


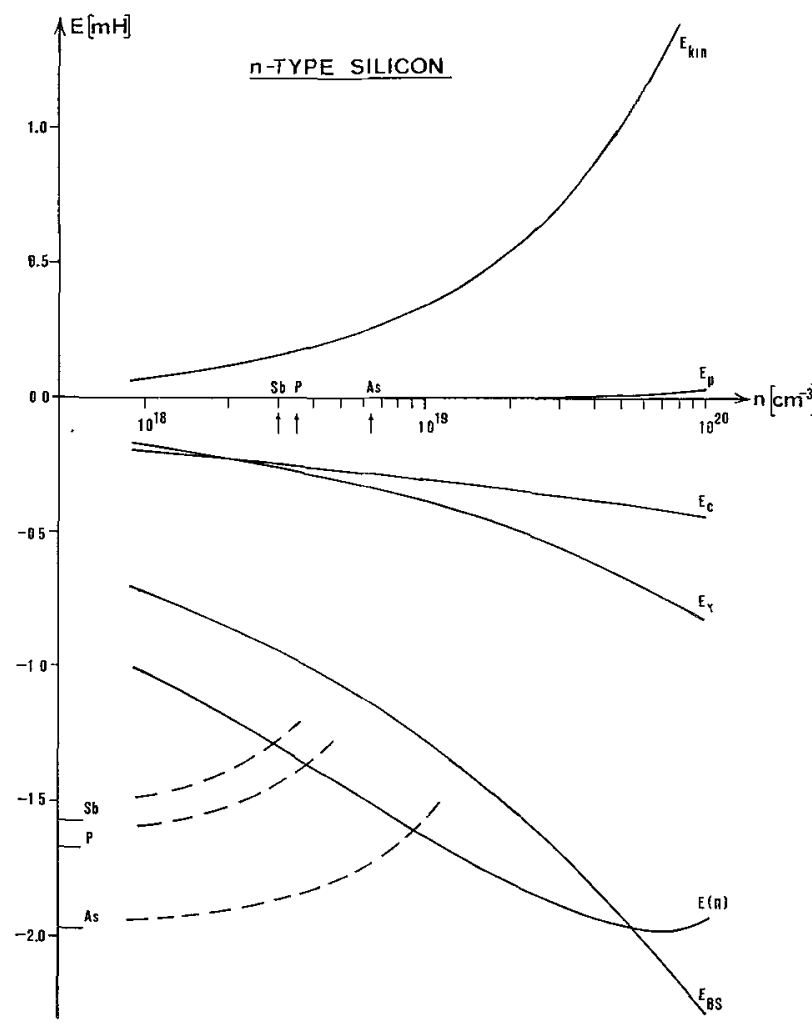

FIG. 1, - The fulldrawn curves represent the various contributions to the energy of the metallic phase of n-type Si as explained in the text. The dashed curves refer to the energy of an electron bound to a donor ion. The ionization energies for isolated impurities indicated on the vertical axis are from [28]. Experimental critical concentrations from [21] are indicated on the horizontal axis. Energies are given in milli-Hartree $(\mathrm{mH})$.

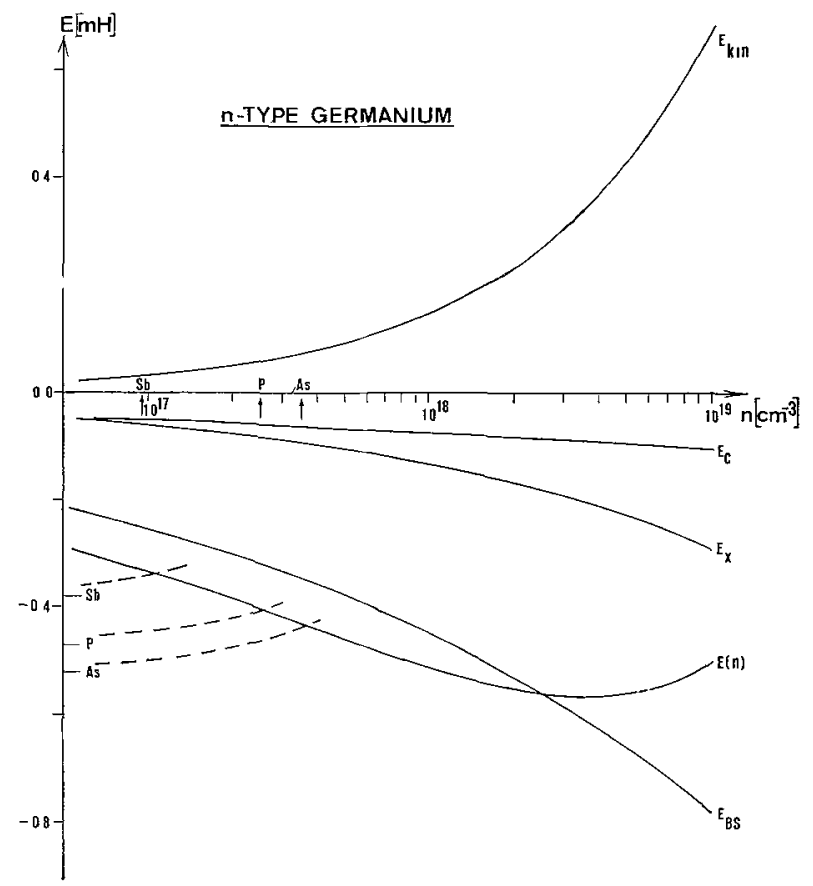

FIG. 2, - The notations are the same as in figure 1, but for n-type Ge. Experimental values of $n_{c}$ are from [1]. should therefore ask ourselves if the present results are fortuitous. A comparison of the energies of two phases as in figures 1 and 2 is for example always sensitive to small errors in one or both of the energy curves. In particular the variation of $I$ was calculated in a rather rough way. Furthermore one may expect that third order corrections in $E_{\mathrm{BS}}$ are important since the perturbing potential is essentially a Coulomb potential. Besides investigating these different approximations more carefully the model should be tested against other cases as well. It is for example evident that the model can easily be extended to p-type semiconductors. Also the model should be used to compute quantities like specific heat and spin susceptibilities of the metallic phase which are available from experiments in order to obtain a more crucial test.

In view of the present results we may briefly address ourselves to the question whether the observed MNMtransition in uncompensated $\mathrm{Ge}$ and $\mathrm{Si}$ is dominated mainly by disorder or by electron correlation (or rather cohesive properties in our model). The fact that our model is based on not too unrealistic assumptions seems to indicate, together with previous models discussed in the introduction, that the latter mechanism in the most important one. We again stress, however, that our good results may be accidental.

Finally, some remarks could be made about the general character of the energy curves in figures 1 and 2 . In the case of for example metallic fluids or electronhole systems the intersection of two energy curves signals a two-phase region. A system then gains in cohesive energy by forming droplets or metallic islands. In doped semiconductors the impurity atoms in general are not free to move, however, so such a formation of islands would occur at the price of the formation of differently charged regions. If on the other hand there would be cases where the impurity atoms are mobile a condensation into metallic droplets is to be expected.

Note added in proof: Above we have considered the critical concentration for multivalley semiconductors like Ge and Si. R. Romestain, S. Geschwind, and G. E. Devlin ( $J$. Physique, this issue) report data for In in CdS which has only a single isotropic conduction band minimum. The experimental estimate is $n_{\mathrm{c}}=2.2( \pm 0.2) \times 10^{17} \mathrm{~cm}^{-3}$. With $I=27 \mathrm{meV}$, $m^{*}=0.20$, and $\kappa=8.42$ (SArToH, M., J. Phys. Soc. Japan 21 (1966) 2540 ; Verleur, H. W., BARKER, A. S., Phys. Rev. 155 (1967) 750) our model yields $2.4 \times 10^{17} \mathrm{~cm}^{-3}$ in close agreement with experiments. If, on the other hand, we choose $\kappa=9.0$ (MAHAN, G., in Physics and Chemistry of II-VI Compounds, Aven, M. and Prenner, J., Eds. (Wiley, New York) 1967) the calculated value is $3.7 \times 10^{17} \mathrm{~cm}^{-3}$ which indicates the sensitivity of model to the choice of $\kappa$. For $\mathrm{CdS}: \mathrm{Cl}$ a MNM-transition has been observed to take place somewhere between $5 \times 10^{17}$ and $1.6 \times 10^{18} \mathrm{~cm}^{-3}$ (ADAMS, F. D., Look, D. C., Brown, L. C. and Locker, D. R., Phys. Rev. B 4 (1971) 2115). With 
$I=32 \mathrm{meV}, m^{*}=0.20$ and $\kappa=8.42$ our model gives $5.6 \times 10^{17} \mathrm{~cm}^{-3}$.

As regards the possibility of a condensate of impurity atoms contemplated above recent experiments (Kastallski, A. A. and Maltsev, S. B., Solid State Commun. 17 (1975) 107 ; Vengalis, B. Yu., KastallSki, A. A. and Maltsev, S. B., PISMA, Zh. Eksp. Teor.
Fiz. 22 (1975) 7 (JETP Lett. 22 (1975) 3)) and theoretical work (Rose, Jr., J. H., SHORE, H. B. and Zaremba, E., Phys. Rev. Lett. 37 (1976) 354) seem to indicate that metallic islands are indeed formed in $\mathrm{Si}$ heavily doped with $\mathrm{Li}$ and at elevated temperatures. Our calculated equilibrium density (Fig. 1) is in reasonable agreement with the estimate of Rose $e t$ al.

\section{References}

[1] For a general survey, see

Motr, N. F., Metal-insulator transitions (Taylor \& Francis Ltd., London) 1974.

[2] Hubbard, J., Proc. R. Soc. A 276 (1963) 238 ; A 281 (1964) 401.

[3] Anderson, P. W., Phys. Rev. 109 (1958) 1492.

[4] Mikoshiba, N., Rev. Mod. Phys. 40 (1968) 833.

[5] Cohen, M. H. and JorTner, J., Phys. Rev. Lett. 30 (1973) 699.

[6] Rogers, F. J., Graboske, H. C. and Harwoon, D. J., Phys. Rev. A 1 (1970) 1577.

[7] Lam, C. S. and Varshni, Y. P., Phys. Rev. A 4 (1971) 1875. (This article contains a large number of references to earlier works on the TF screened Coulomb potential.)

[8] Krieger, J. B. and Nightingale, M., Phys. Rev. B 4 (1971) 1266.

[9] Martino, F., Lindell, G. and Berggren, K. F., Phys. Rev. B 8 (1973) 6030.

[10] Sinha, O. P. and Puri, O. P., Phys. Rev. B 12 (1975) 1395.

[11] AlmbladH, C.-O., von Barth, U., Popovic, Z. D. and StotT, M. J., Phys. Rev. $B$ (in press).

[12] Caron, L. G. and Kemeny, G., Phys, Rev. B 3 (1971) 3007.

[13] Berggren. K. F., Phil. Mag. 27 (1973) 1027.
[14] Bergersen, B., Rostworowski, J. A., Eswaran, M., Parsons, R. R. and Jena, P. (to be publ.).

[15] Gutzwiller, M. C., Phys. Rev. 137 (1965) A 1726.

[16] Berggren, K. F., Phil. Mag. 30 (1974) 1.

[17] Chao, K. A. and Berggren, K.-F., Phys. Rev. Lett. 34 (1975) 880 .

[18] Frood, G. D. H., Proc. Phys. Soc. London 75 (1960) 185.

[19] Bergaren, K.-F., J. Chem. Phys. 60 (1974) 3399 ; 61 (1974) 2989.

[20] AoKi, H. and Kamimura, H., J. Phys. Soc. Japan 40 (1976) 6.

[21] Castner, T. G., Lee, N. K., CietoszyK, G. S. and SalinGER, G. L., Phys. Rev. Lett. 34 (1975) 1627.

[22] Мотт, N. F., The degenerate electron gas in tungsten bronzes and in highly doped silicon (in manuscript).

[23] Berggren, K.-F. and Sernelius, B., Solid State Commun. 19 (1976) 487.

[24] Pokrovskir, Ya, Phys. stat. sol. (a) 11 (1972) 385.

[25] Baltensperger, W., Phil. Mag. 44 (1953) 1355.

[26] Pantelides, S. T., Proc. 12th Int. Conf. semicond., Ed. Pilkuhn E. (Teubner, Stuttgart) 1974.

[27] BRINKMan, W. F. and RICE, T. M., Phys. Rev. 7 (1973) 1508.

[28] Bassani, F., Iadonisi, G. and Preziosi, B., Rep. Prog. Phys. 37 (1974) 1099. 